\title{
Measurements and Modelling of Radar Signatures of Large Wind Turbine Using Multiple Sensors
}

\author{
Waleed Al-Mashhadani, Anthony \\ Brown, Laith Danoon \\ School of Electrical \& Electronic \\ Engineering \\ University of Manchester, \\ Manchester, UK
}

\author{
Colin Horne, Riccardo Palamà, \\ Hugh Griffiths \\ Department of Electronic \& \\ Electrical Engineering \\ University College London, \\ London, UK
}

\author{
Jarez Patel, Francesco Fioranelli \\ School of Engineering \\ University of Glasgow \\ Glasgow, UK
}

\begin{abstract}
This paper presents initial results on the characterization of radar signatures of wind turbines, in particular larger wind turbines (capacity over $7 \mathrm{MW}$ ) used for offshore wind farms. Experimental results from simultaneous data collected using a passive DVB-T (Digital Video Broadcasting-Terrestrial) radar sensor and an active radar working at S-band are presented, as well as some comments on the parallel work on the modelling of the turbine and on the development of detection algorithms specific for this type of clutter. The initial results show significant variability of the signatures for different radar sensors used, but also for different parameters (e.g. polarization) for the same radar sensor and operational conditions of the turbine (rotation speed, yaw angle).
\end{abstract}

Keywords-Wind Farm Clutter, EM Modelling, Radar Signatures, Multistatic Radar, Passive Radar

\section{INTRODUCTION}

The negative effect of wind farm on the performance of radar systems has been widely reported, especially for critical applications such as those for air traffic control, weather radar, and airspace surveillance radar [1]. These effects include shadowing and masking of targets of interest, both in the time domain due to large Radar Cross Section (RCS) of the turbines and in the frequency domain, due to the Doppler shifts induced by the rotating movements of the blades. As an effect of this, the development of wind farms - which is a priority to reduce reliance on fossil fuels - can be hindered by "radar objections" from defense or civil aviation authorities. Research within the radar community has been undertaken to characterize the radar signatures of wind turbine and farms, both from a modelling perspective [2-5] and from an experimental standpoint [6-11], as well as looking at improving signal processing and detection algorithms to make existing radar systems more resilient to wind farm clutter.

In this paper, we aim to present initial results of a collaborative project carried out within the UK SUPERGEN (research hub for wind energy). The objective is characterizing the radar signature of large $(>7 \mathrm{MW})$, offshore wind turbines, which are significantly larger and including more individual turbines than their onshore counterparts [12]. This is particularly significant in the UK, where recent governmental policies have promoted the development of large offshore wind farms across the coasts of Britain. The work will combine modelling of the RCS of these structures in different scattering and geometry regimes, alongside experimental data from diverse radar sensors such as Passive Bistatic Radar (PBR) and bistatic/multistatic radar. PBR has been receiving significant interest as a 'fill-in' sensor for air surveillance purposes, particularly as a technology to reduce the degradation caused by wind farms. The effects of wind turbines on the operation of radar is investigated in [1], where it is estimated that a $60 \mathrm{~m}$ mast turbine illuminated by a $1 \mathrm{~m}$ wavelength is expected to have RCS of the order of $45 \mathrm{dBm}^{2}$, some $15 \mathrm{~dB}$ larger than a typical aircraft. Offshore turbines are significantly larger, hence exhibit a greatly larger RCS. Simulation of a WiMAX based passive radar for marine surveillance is described [14], including the detection coverage near windfarms. [15-17] discuss the PARASOL collision warning system based on DVB-T transmissions (Digital Video Broadcasting-Terrestrial). This system is designed to work within a turbine park with the function of detecting approaching aircraft, such that collision avoidance lights can be activated only as necessary, avoiding constant light pollution, affecting both humans and attracting undesired bird life to the area. Kulpa et al. [18] provide an analysis, through simulation and experimentation, of the influence of wind turbines on Digital Video Broadcasting Terrestrial (DVB-T) based passive radar systems. A wide range of different turbine sizes are considered, including one of roughly comparable dimensions (164 $\mathrm{m}$ diameter) to the one measured in this work $(174 \mathrm{~m})$, although the dimensions of the experimentally measured turbine are not stated. Multistatic/distributed radar systems have also been proposed to address this wind farm clutter issues, assuming that the additional spatial diversity from the network of radar sensors may be advantageous to reduce the clutter-to-target cross section and the spread in Doppler of the undesired clutter signal [10-11].

The rest of this paper is organized as follows: Section II discusses the preliminary turbine modelling work undertaken, Section III presents the experimental configuration of both the monostatic and PBR systems, Section IV explores the data analysis methods and presents gathered results; finally Section $\mathrm{V}$ concludes the paper and reviews the next steps. 


\section{MODELLING WORK}

This work adapts the Rectangular Meshing for RCS Approximation (ReMeRA) modelling methodology of the wind farm RCS modelling tool that was developed at the University of Manchester [19]. The adapted modelling tool devised a methodology that gives an accurate geometrical representation of the wind turbine for RCS and Doppler modelling. This model was based on Physical Optics (PO) approximation and extensively tested against measured RCS data of canonical shapes with flat and curved surfaces [19 20]. Additionally, The ReMeRA was also compared against the RCS modelling results obtained from FEKO [21] for a full wind turbine blade [22]. The good agreement with the measurements and results from other models gives confidence that the ReMeRA model can predict the RCS of turbine components to a good level of accuracy. The RCS of a turbine can be very large based on the large size of the turbine structure and depending on the turbine orientation with respect to the radar. The RCS modelling for this type of structure is a complex parameter and depends on incident wave, frequency, material, illumination pattern and distance from the source [22]. Additionally, the complexity in wind turbine geometry and the extension over hundreds or thousands of wavelengths at radar frequencies proved to be a challenging task [19]. However, the new methodology (ReMeRA) achieved the modeling of turbine's RCS in computationally efficient manner that can be used on standard desktop computers.

\section{A. Modelling tool process and assumptions:}

The modelling tool divides the turbine tower and blades into segments of small sections along their length, as it is illustrated in the 3D model shown in Fig. 1. A single blade is typically divided into number of sections between 100 and 200. The number of sections for the tower is approximately twice the number of segments used in the blade. However, segments number and size can vary for different applications [19]. The model computes the RCS for each segment and considers it as a potential target that creates its own radar signature. This radar return is modeled by accounting for the antenna pattern and other propagation factors to ensure good representation of the environment as well as the turbine geometry.

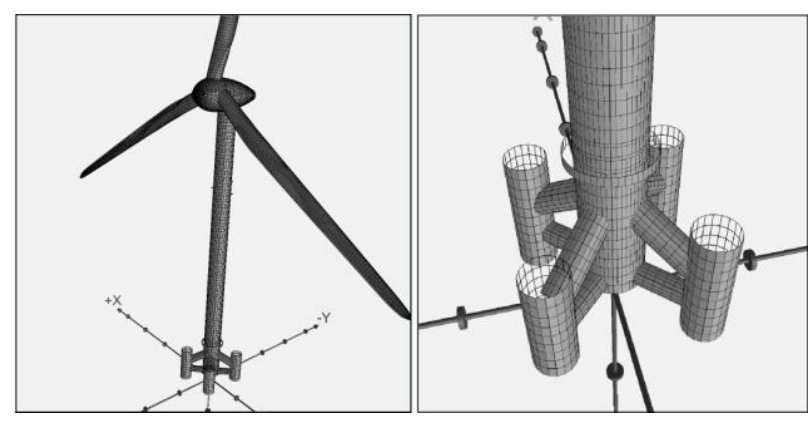

Fig. 1. Wind Turbine 3D illustration of the RCS modeling tool
The complex aerodynamic profile of the blade makes the RCS modelling highly variable with time, depending greatly on the incidence angle of the radar beam. It was important to represent accurately the blade geometry for better RCS modelling, because a small change in the profile at a radar frequency will result in a major change in the RCS profile of the blade -this is also true when blade bending is experienced due to wind loading. In comparison to the tower return, the nacelle component has much smaller return due to its smaller size. The radar return from the nacelle can be significant when the direct incident wavefront illuminates the flat plates from the side of the nacelle, increasing the RCS. Nacelle's with a round design tend to have higher RCS profile than the rectangular design [23]. Turbine components can shadow each other for a short period, this is referred to as self-shadowing. The adapted RCS model does not account for self-shadowing, because it showed no significant impact on the RCS modelling based on the short period and small shadowing area. The selfshadowing is referred to as "RCS chopping" [24]. Wind loading and solar heating also affect the tilt and bend of the turbine tower and blades. The tower component can bend up to $0.4 m$ at the top sections under maximum wind load [19]. In addition, the differential expansion of the tower due to the solar heating of one side can cause similar effect on the tower [19]. This bending change in tower posture is not accounted for. It might effect on the RCS modelling when signal reflects of the bended part away from radar sensors. With these impact factors in mind, the current model validates the assumption according to the field experiment scenario, that the blade and tower to be straight and bend free. The model accounts for the far-field and nearfield RCS modelling [19]. This aspect is very important when it comes to multistatic radar RCS modeling of a wind turbine. The radar system includes multiple receivers that are located at different sites. As such, some sensors can be in the far-field and others can be located within the nearfield, depending on the antenna used and the wavelength.

\section{B. Modeling results:}

Results from the simulation tool are shown in Fig. 2 and 3; the power received and the wind turbine RCS for the monostatic radar scenario (NetRAD, see section III). The transmitted power was set to $23 \mathrm{dBm}$, operating frequency at $2.4 \mathrm{GHz}$ and the distance between the wind turbine and radar sensors was setup to be $300 \mathrm{~m}$, corresponding to location $\mathrm{E}$ in Fig. 4. The simulation results show an increase in RCS and power received figures due to the large reflective tower and the visibility of the side of the nacelle component. The plot in Fig. 2 shows a periodic pattern in the radar signature of power received due to the reported blades positions for each $120^{\circ}$ rotation. This is clearly illustrated from the RCS values that correlate with the power received. Fig. 3 illustrates the RCS modeling of the wind turbine from the prospect of different radar range and blade rotation. At a range of $300 \mathrm{~m}$, the model shows a constant RCS value that is dominated from the turbines' base and tower, varying by $\pm 100 \mathrm{~m}$ about $300 \mathrm{~m}$. The blades' RCS modeling is shown noticeably and the pattern is periodic with each $120^{\circ}$ rotation. 


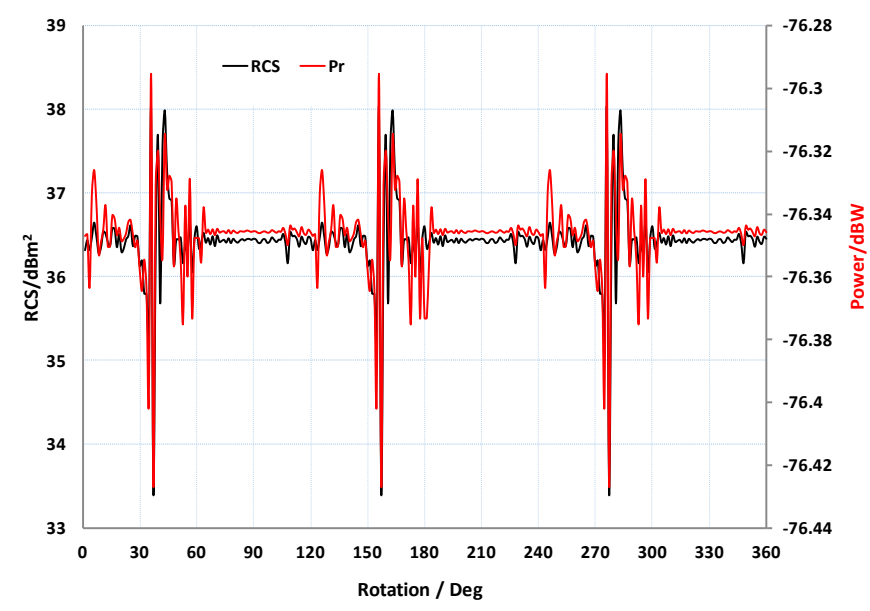

Fig. 2. Wind turbine RCS vs Power Received (monostatic NetRAD). Wind direction $70^{\circ}$

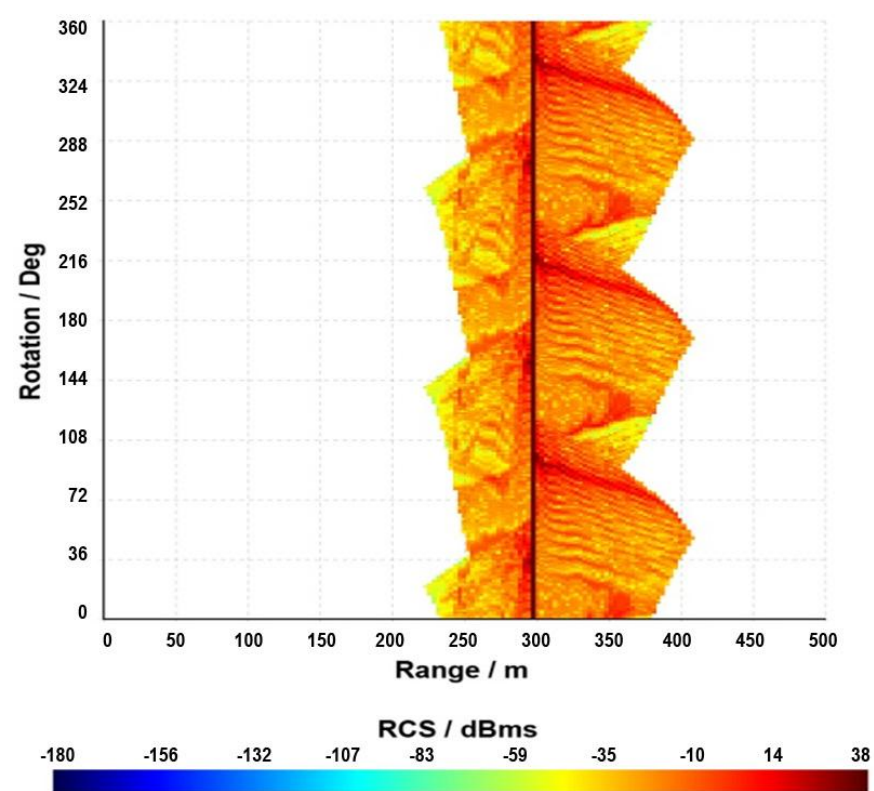

Fig. 3. Wind turbine RCS (monostatic NetRAD). Wind direction $70^{\circ}$

\section{EXPERIMENTAL CONFIGURATIONS \& SETUP}

\section{A. Wind Turbine Trial Geometry}

Fig. 4 shows the location of the experimental radar measurements, performed at the Leven site of the UK ORE Catapult in Scotland, in October 2017. The location of the turbine is denoted as point $\mathrm{A}$, just next to the shore, whereas points B-E indicate the location of the active and radar sensors throughout the two days of experiments. The turbine is a $7 \mathrm{MW}$ Samsung model, with rotor diameter equal to $171.2 \mathrm{~m}$, height of the hub $110.6 \mathrm{~m}$ and length of each of the 3 blades equal to approximately $85.6 \mathrm{~m}$. The turbine is designed to operate with wind speed between 3.5 and $25 \mathrm{~m} / \mathrm{s}$, generating rotation speed of approximately 5.9 to $10.6 \mathrm{rpm}$, according to the information supplied by ORE-C, who maintains this turbine. It is important to notice that this model of turbine, designed for an offshore usage, is much larger than the onshore variants whose radar signatures were measured in previous work [6-11]. Groundtruth data from ORE-C (rotation velocity and yaw angle of the turbine over time) will allow correlating the passive and active radar signatures with the actual behavior of the turbine, enabling a step forward compared to previous work where this data was not made available by the wind farm management [10-11].

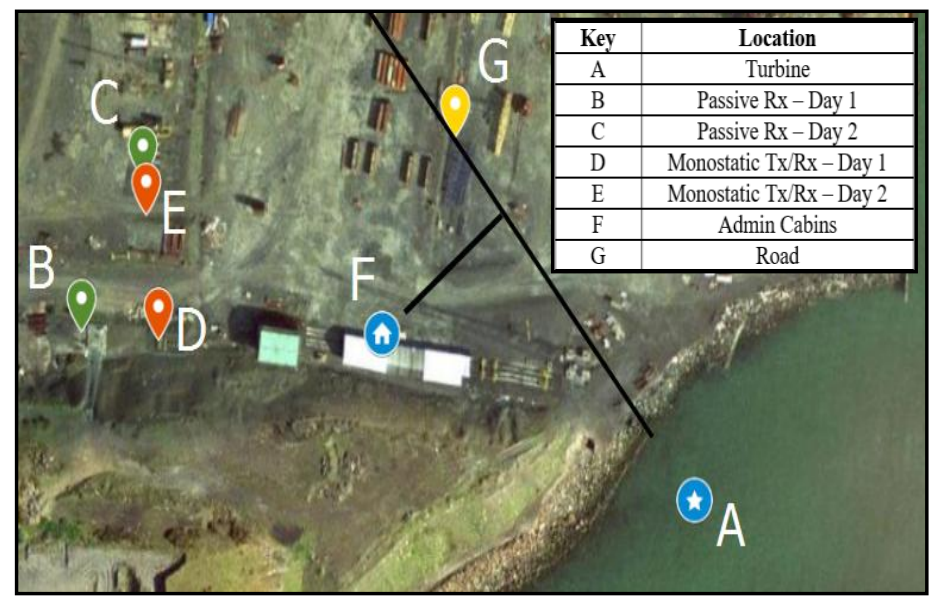

Fig. 4. Layout of the experimental site at the ORE Catapult site, Leven, Scotland, UK

\section{B. Passive Bistatic Radar}

The passive setup employs the Craigkelly DVB-T transmitter located approximately $17 \mathrm{~km}$ to the south-west of the turbine site. The transmitting tower is $125 \mathrm{~m}$ in height, and has a transmit power of $70 \mathrm{dBm}$ for the COM5 service; this is the channel to be utilized for the measurements. The reference antenna is directed towards the DVB-T transmitter, and the surveillance antenna towards the target scene. The surveillance antenna is physically shielded from the direct signal by suitable placement of the trials vehicle. Measurements were recorded from two different receiver locations, providing bistatic angles of approximately $53^{\circ}$ and $65^{\circ}$. This is illustrated for the day 2 configuration in Fig. 4, locations B and C. Fig. 5 shows the turbine as viewed from the passive surveillance antenna on day 2, location $\mathrm{C}$.

The PBR receiver is formed from two standard COTS digital TV aerials, and a dual channel Software Defined Radio (SDR), the LimeSDR. The device communicates with a laptop computer through a direct USB3 connection, which runs the proprietary Graphical User Interface (GUI) software, named LimeSuite. To allow observation of the received signals, Python scripts where employed to extract and record the data from the GUI. Each receive channel samples 12 bit-complex data at 146.3 MSPS, which is then decimated through hardware by a factor of 16 . This provides a final complex data rate of 64/7 MSPS or 9.143 MSPS, which delivers the desired 8192 samples per DVB-T symbol. Data sets were collected with a duration of 5, 10 or 20 seconds, this is then stored on a hard disk for offline analysis. Fig 6 shows an example of part of the LimeSuite display: the blue trace illustrates the direct received signal, the red being the surveillance signal amplified by an additional $12 \mathrm{~dB}$ of gain above the reference channel, provided through an internal LNA. The center frequency is set to 666 
$\mathrm{MHz}$ and each DVB-T channel occupies $8 \mathrm{MHz}$ including a $200 \mathrm{kHz}$ guard zone at each end.

\section{Active Radar}

The active radar utilized, is the multistatic radar,NetRAD developed at University College London over the past years. It is an S-band, coherent, pulse-Doppler radar, operating at a carrier frequency of $2.4 \mathrm{GHz}$. For the experiments reported in this paper, we used $23 \mathrm{dBm}$ transmitted power, $1 \mu$ s duration and $45 \mathrm{MHz}$ bandwidth of the linear chirp modulation of the pulse, and $5 \mathrm{kHz}$ Pulse Repetition Frequency (PRF), enough to obtain unambiguous Doppler signatures. The antennas used were dual-polarized dish antennas, with $18^{\circ}$ (horizontal) $\times 19^{\circ}$ (vertical) beam-width and $18 \mathrm{dBi}$ gain. Due to technical issues, only suitable monostatic data were recorded, at two locations indicated in Fig. 4 (locations $\mathrm{D}$ and $\mathrm{E}$ ) separated by approximately $70 \mathrm{~m}$ from each other and with distance to the turbine being roughly $300 \mathrm{~m}$. For each location, multiple recordings of 1 minute each were taken, repeating the measurements with all the four possible polarization combinations.

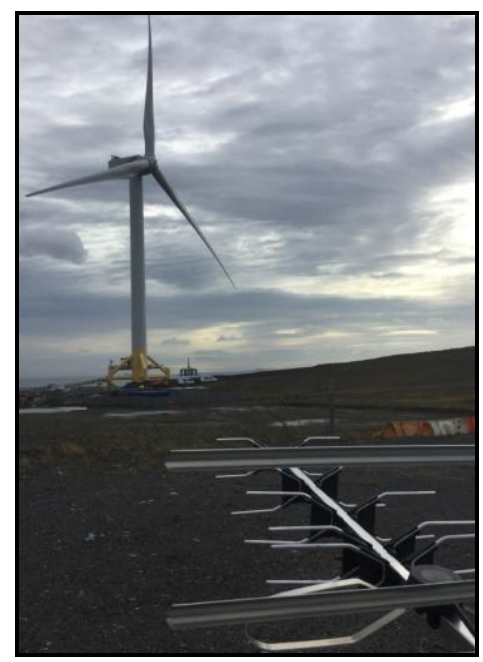

Fig. 5. ORE-C turbine as seen from the passive radar receiver site

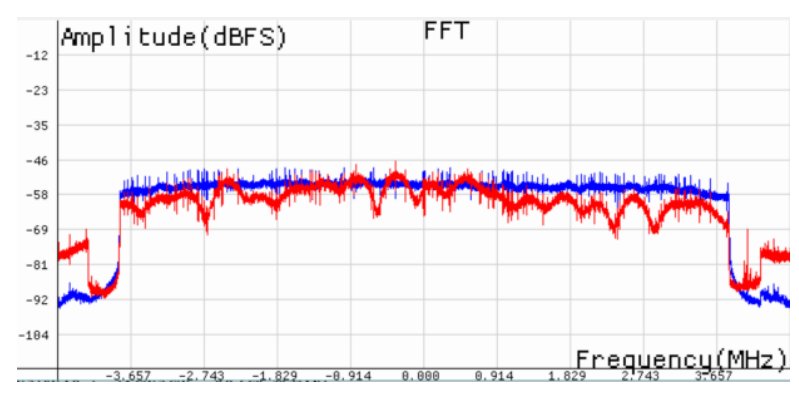

Fig. 6. Spectrum of passive reference and surveillance signals

\section{INITIAL RESULTS}

\section{A. Passive Radar Data}

The data from the reference channel is processed to identify the start of the first full DVB-T symbol [25]. Using this knowledge, the symbols are extracted into an array, discarding the cyclic guard added to the symbol that serves as a multipath measure. As the data recorded from the two channels of the SDR are synchronized in time, the same offsets can also be applied to the surveillance channel, but in this case an additional number of samples are maintained equal to the number of range cells to be processed. This value is set to 50 in the results shown. The surveillance channel data is pulse compressed by cross correlation using the reference channel data from the corresponding symbol. The required 50 range cells of data are extracted for further processing.

Several plots are shown here to present some preliminary results from the processing of PBR results. Unless otherwise stated, data is captured in a co-polar configuration. Fig. 7 illustrates the spectra of two datasets evaluated over the complete data capture period. The Doppler spread of the raised region in (a) being approximately $-145 \mathrm{~Hz}$ to $+130 \mathrm{~Hz}$, and in (b) $-240 \mathrm{~Hz}$ to $230 \mathrm{~Hz}$. Trials logs recorded that the turbine face appeared to be directed more towards the receiver, and rotating more slowly in data capture (a). Fig. 8 (a) and (b) show results from applying the short time Fourier transform (STFT) to the same two datasets, with a Hamming window of approximately $0.25 \mathrm{~s}$. The FFT overlap is approximately $97 \%$. The typical pattern of Doppler flashes expected for a rotating turbine can be seen. The turbine consists of a three bladed rotor; hence, the period of three flash intervals corresponds to a full $360^{\circ}$ rotation of the turbine. Fig. 8 (a) and (b) show rotation rates of $8.8 \mathrm{rpm}$ and $10.3 \mathrm{rpm}$ respectively. Fig. 8(c) shows the STFT result for data captured from a cross polarized configuration. This data represents a rotation rate of $8.8 \mathrm{rpm}$.
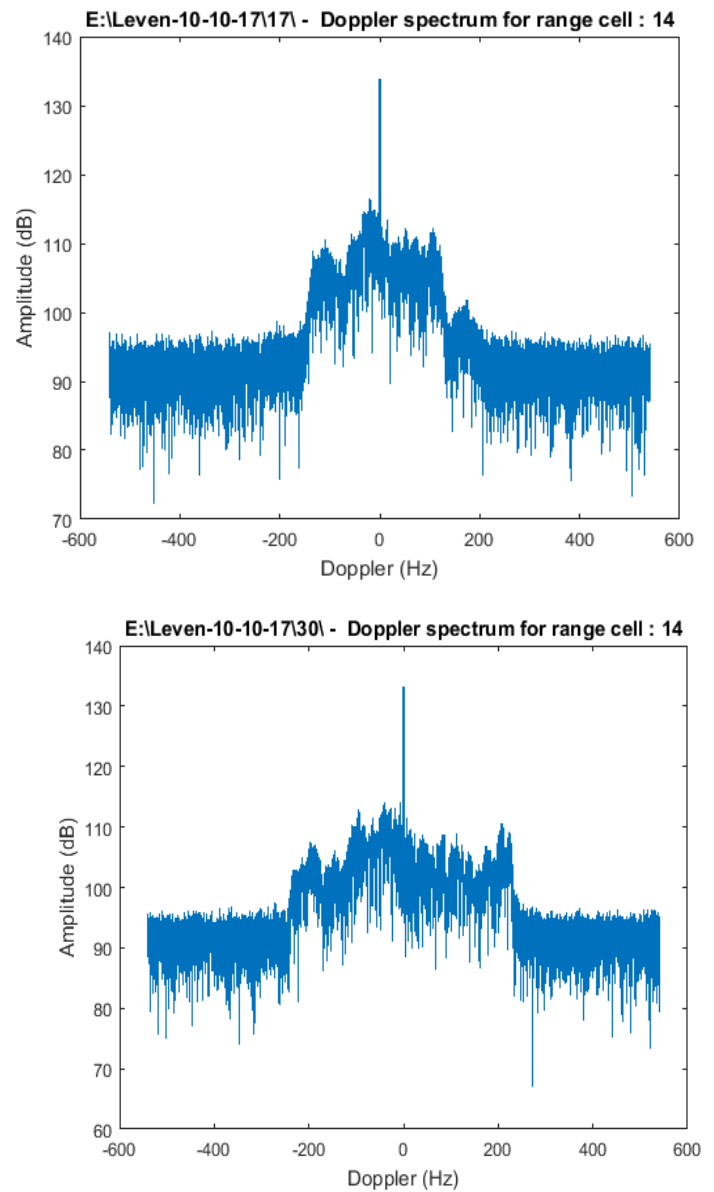
Fig. 7. Doppler spectra examples as recorded by PBR sensor
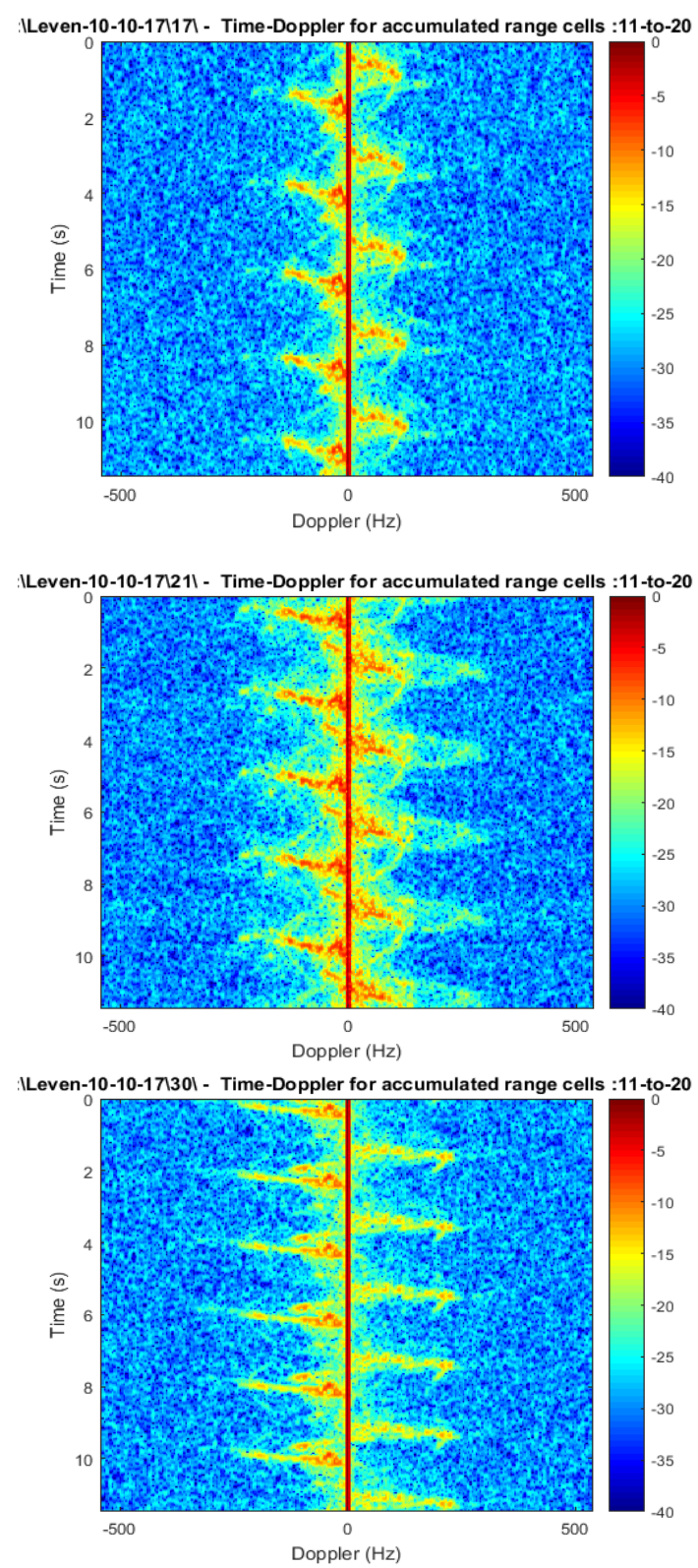

Fig. 8 Spectrogram examples as recorded by the PBR sensor: (a) and (b) two examples of co-polarised data, (c) an ecample of cross-polarised data

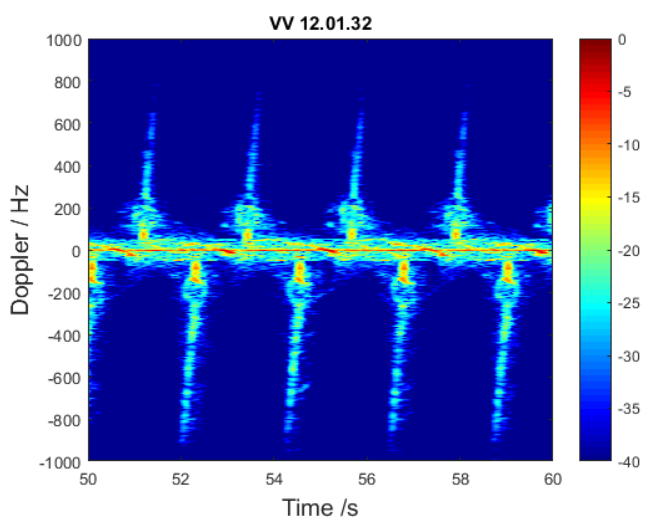

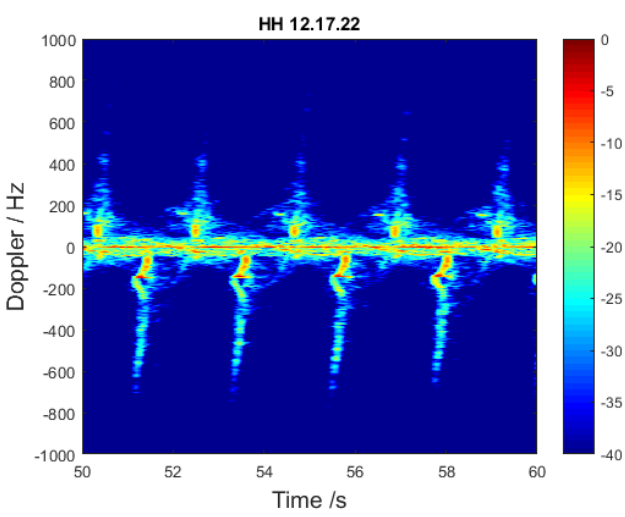
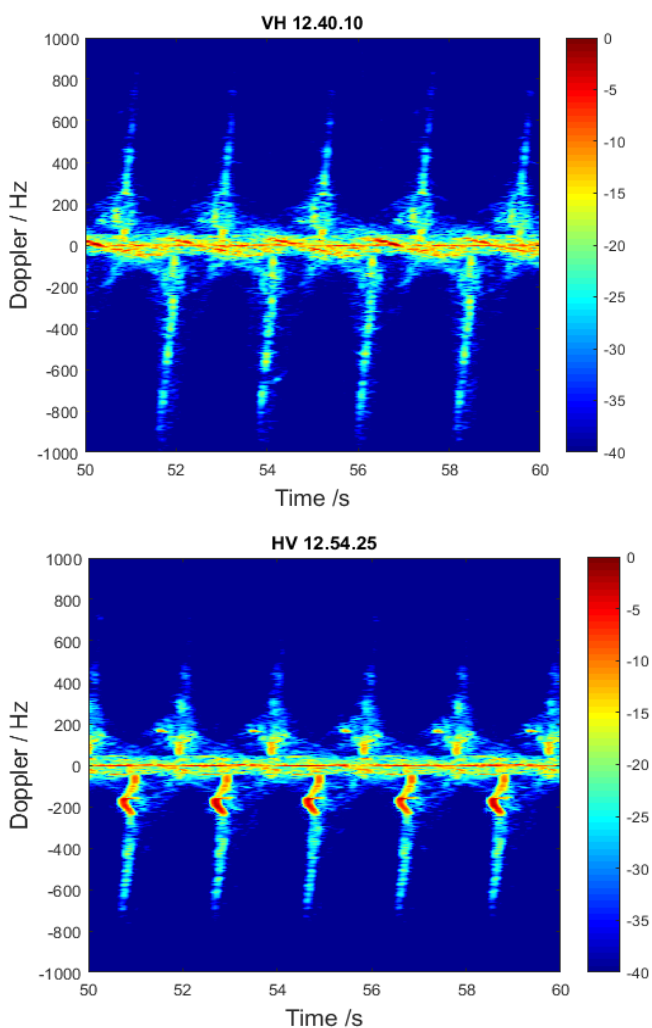

Fig. 9 Four examples of spectrograms (micro-Doppler signature) of the wind turbine recorded by active $\mathrm{S}$-band radar at four polarizations

Fig. 10 shows an example of 4 Probability Density Function (PDFs) of the amplitude samples of the return from the wind turbine, for the four different polarizations considered in the previous figures. In this case, only one range bin has been considered for each measurement to calculate the PDF, in particular the range bin with the highest return, which presents "flashes" of higher intensity over time due to the rotation movement of the blades. The different polarizations appear to provide different PDFs shapes in terms of average intensity value and different width of the tail of the distribution (related to the high intensity events). More investigation is needed to characterize how these PDFs change for different polarizations and for different operational conditions over time for a given polarization. 


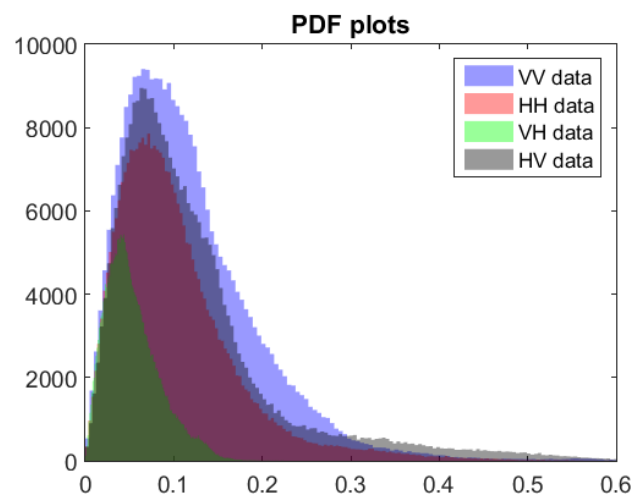

Fig. 10 PDFs plots of the amplitude samples of the wind turbine return for different polarisation combinations

\section{CONCLUSION}

This paper has presented initial results regarding measurements and modelling of the radar signatures of large wind turbines used for offshore wind farms. The aim of the project is to record these signatures with different radar sensors, exploring the effect of polarization and frequency diversity, and compare simultaneous passive and active radar measurements. These results were obtained using an active monostatic S-band radar and a DVB-T passive radar. Future work will aim to correlate modelling and experimental results with the ground truth data (rotation speed, turbine orientation over time) from the turbine management to better understand their impact on the radar signatures. Further experimental data will be collected, with more sensors (bistatic recordings at $\mathrm{S}$ band, additional frequency bands) and at different geometries with respect to the turbine. Wind-turbine clutter has a detrimental effect on radar performance in detecting and tracking targets, in particular aircraft. A critical challenge consists of small aircraft flying near a wind turbine, whose moving blades can be erroneously confused as targets, then inducing false alarms and tracks. If the aircraft has itself a micro-Doppler signature (as for helicopters or drones), it is very important to distinguish the wind-turbine scattering from the target's, in order to detect and classify the aircraft. Future developments will involve testing and validating novel detection and tracking algorithms in the presence of windturbine clutter, possibly injecting synthetic targets in the real data presented in this work.

\section{ACKNOWLEDGMENTS}

The authors acknowledge financial support from SUPERGEN Flexible Funding 2 (EPSRC EP/L014106/1), logistic support from Peter and Lorna at ORE Catapult, and support from industrial partners (Prof C. Baker at Aveillant and RPS Energy on behalf of DONG Energy).

\section{REFERENCES}

[1] C. A. Jackson, "Windfarm characteristics and their effect on radar systems," 2007 IET International Conference on Radar Systems, pp. 1-6, 15-18 October, Edinburgh, UK.

[2] L. R. Danoon and A. K. Brown, "Modelling methodology for computing the radar cross section and Doppler signature of wind farms," IEEE Transactions on Antennas and Propagation, vol. 61, 2013.
[3] L Danoon, A Brown, 'Radar cross-section analysis of wind turbine blades with radar absorbing materials', European Radar Conference (EuRAD) 2011, pp 97 - 100. October 2011, Manchester.

[4] O. Karabayir et al, "Wind turbine signal modelling approach for pulse Doppler radars and applications," IET Radar, Sonar \& Navigation, vol. 9, pp. 276-284, 2015.

[5] F. Weinmann and J. G. Worms, "EM scattering effects caused by wind turbines," 2016 10th European Conference on Antennas and Propagation (EuCAP), Davos, 2016, pp. 1-5.

[6] B. M. Kent, K. C. Hill, A. Buterbaugh, G. Zelinski, R. Hawley, L. Cravens, et al., "Dynamic Radar cross section and radar Doppler measurements of commercial General Electric windmill power turbines Part 1: predicted and measured radar signatures," IEEE Antennas and Propagation Magazine, vol. 50, pp. 211-219, 2008.

[7] A. Buterbaugh, B. M. Kent, K. C. Hill, G. Zelinski, R. Hawley, L. Cravens, et al., "Dynamic radar cross section and radar Doppler measurements of commercial General Electric windmill power turbines Part 2: predicted and measured Doppler signatures," 2007 Antenna Measurements Techniques Association (AMTA) Symposium.

[8] H. J. de Wind, K. H. Kloke, U. Böniger, H. Pratisto, 'Comparison of the recorded RCS and spectra of three different wind turbines on L- and Sband', 2015 IEEE Radar Conference, Johannesburg, 2015, pp. 266-271.

[9] O. A. Krasnov and A. G. Yarovoy, "Polarimetric micro-Doppler characterization of wind turbines," 2016 10th European Conference on Antennas and Propagation (EuCAP), Davos, 2016, pp. 1-5.

[10] F. Fioranelli, M. Ritchie, A. Balleri, H. Griffiths, 'Experimental analysis of multistatic multiband radar signatures of wind turbines', IET Radar, Sonar \& Navigation, vol. 10(8), 1400-1410, October 2016.

[11] F. Fioranelli, M. Ritchie, A. Balleri, H. Griffiths, 'Practical investigation of multiband bistatic and monostatic radar signatures of wind turbines, IET Radar, Sonar \& Navigation, vol. 11 (6), 909-921, June 2017.

[12] L. Danoon, W. Al-Mashhadani and A. Brown, "Modelling the impact of offshore wind farms on safety radars on-board Oil and Gas platforms," 2016 10th European Conference on Antennas and Propagation (EuCAP), Davos, 2016, pp. 1-5.

[13] Kuschel, H., Heckenbach, J., Schell, J., and Wissmann, W. Effects of wind power plants on passive radar operation. 2009 International Radar Conference "Surveillance for a Safer World".

[14] Chetty K, Woodbridge K, Guo H, Smith G. Passive bistatic WiMAX radar for marine surveillance. 2010 IEEE National Radar Conference.

[15] Kuschel H. Approaching 80 years of passive radar. 2013 International Conference on Radar.

[16] Heckenbach, J., Kuschel, H., Schell, J., \& Ummenhofer, M. Passive Radar Based Control of Wind Turbine Collision Warning for Air Traffic PARASOL. 2015 16th International Radar Symposium (IRS), Dresden.

[17] Palmer, J., Cristallini, D., Kuschel, H. Opportunities and current drivers for passive radar research. 2015 IEEE Radar Conference, Johannesburg.

[18] Kulpa, J., Baczyk, M., Kurowska, A., Rzewuski, S. Wind Farm Interferences in Passive Coherent Location. 2013 International Conference on Radar..

[19] Laith S Rashid, Impact Modelling of Offshore Wind Farms on Marine Radars, The University of Manchester, 2010.

[20] Jenn, D.C. and M.F. Chatzigeorgiadis, A MatLab physical Optics RCS Prediction Code, IEEE Antennas and Propagation Magazine. Aug 2004.

[21] Davidson, D.B., et al. "Recent progress on the antenna simulation program FEKO". South African Symposium on Communications and Signal Processing. 1998. South Africa.

[22] G. J. Poupart, "Wind Farms Impact on Radar Aviation Interests", BWEA Radar Aviation Interests Report, DTI report number W/14/00614/00/REP, September 2003.

[23] Youssef, N.N., Radar cross section of complex targets. Proceedings of the IEEE, 1989. 77(5): p. 722-734.

[24] Butler, M.M. and D.A. Johnson, Feasibility of Mitigating the Effects of Wind farms on Primary Radar. 2003, Alenia Marconi Systems Limited. 